\title{
Proposal of a touch switch with arbitrary form and arrangement
}

\author{
Shun Inoue $^{\mathrm{a}^{*}}$, Kiyotada Matsura $^{\mathrm{a}}$, Akira Yamawaki ${ }^{\text {aa }}$ \\ Kyushu Institute of Technology,1-1 Sensui-cho,Tobata-ku,Kitakyushu-shi,Fukuoka,804-8850,Japan \\ inoue@boss.ecs.kyutech.ac.jp \\ Abstract \\ Section 3 shows the experimental results and discusses \\ about them. Section 4 concludes our paper.
}

Conventional touch switches are premised on the use of a display or wall or other flat surface. Thus, they cannot be applied to many things with any shapes. In addition, since the traditional touch switch is embedded to a product, the user is unable to change its shape and position after shipment. In this paper, we propose a touch switch whose shape and location can be arbitrarily changed. The user can arbitrarily change the shape, location and the number of switches of the touch switch flexibly. Through the preliminary experiments, we show that our touch switches can indicate the switch touched by finger.

Keywords : touch switch, arbitrary form, arrangement

\section{Introduction}

There are touch switches for detecting a position a human finger touching. Since they can eliminate a mechanical switch, the design of the electronic products can be improved. Especially, for the mobile phones, the digital cameras, the digital portable audio players, and so on, the touch switches may be desirable considering the device size. Conventional touch switches are premised on the use of a display or wall or other flat surface. Thus, they cannot be applied to many things with any shapes. In addition, since the traditional touch switch is embedded to a product, the user is unable to change its shape and position after shipment.

In this paper, we propose a touch switch whose shape and location can be arbitrarily changed. By using a metal film or metal plate as a switch, the shape of the switch can be arranged arbitrarily. The switch of the metal film or metal plate can be placed and attached to various locations. The switches can form the array structure. To this array, arbitrary number of switches can be inserted and removed. Many form of array with many different shaped switched can be realized as the user like.

The rest of the paper is organized as follows. Section 2 describes the overview of the proposed touch switch.

\section{Proposed touch switch}

\subsection{Processing flow}

Fig. 1 shows a processing flow to detect the touched switch. Fig. 2 depicts a device organization to realize our touch switch.

A wave generator generates a sine wave with a high-frequency (typically greater than $1 \mathrm{MHz}$ ) to the detector shown in Fig. 2. When the human finger touches the switch as a metal film or metal plate, a series circuit with a resistor and capacitor is virtually formed in the human body for the AC current. Since the AC current flows through the human body to the ground via the touched switch, the closed circuit is formed. As a result, the detector can measure a voltage caused by the touched switch over the resistor of $100 \Omega$. The detector acquires two output voltage, $V_{1}$ and $V_{2}$ shown in Fig. 2.

To determine which switch is touched, these output voltages are used. To avoid several dependencies of a metal used to the switch and the human characteristic and the effect of environment, the ration of $V_{1}$ to $V_{2}$ is used instead of the voltage values.

Since the detector has two probing point in parallel as shown in Fig. 2, the ratio of $V_{1}$ to $V_{2}$ does not change even if the amount of the current through the resistor changes. 


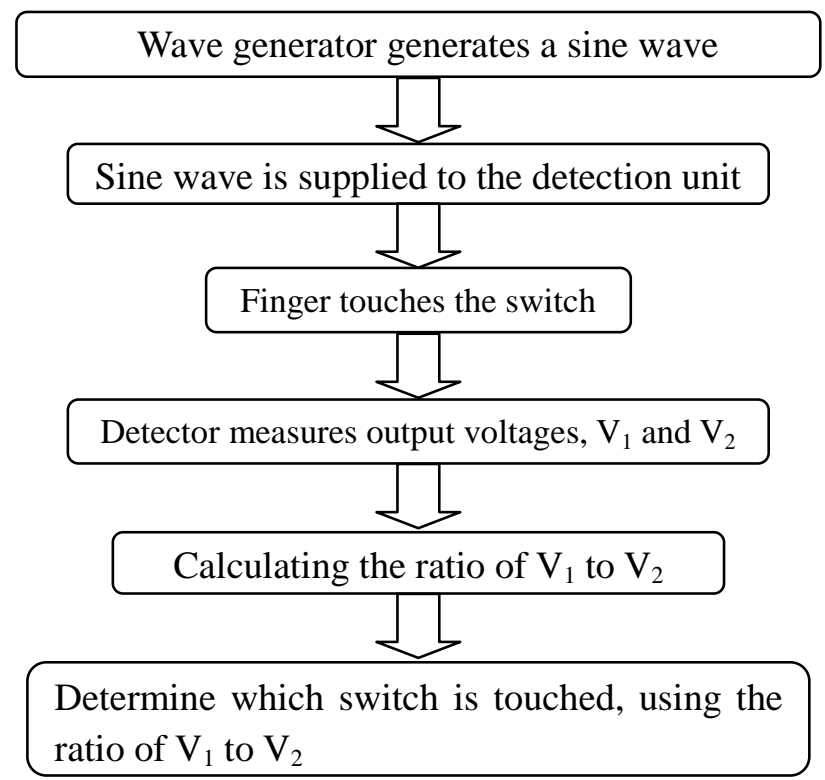

Fig.1 Detecting method
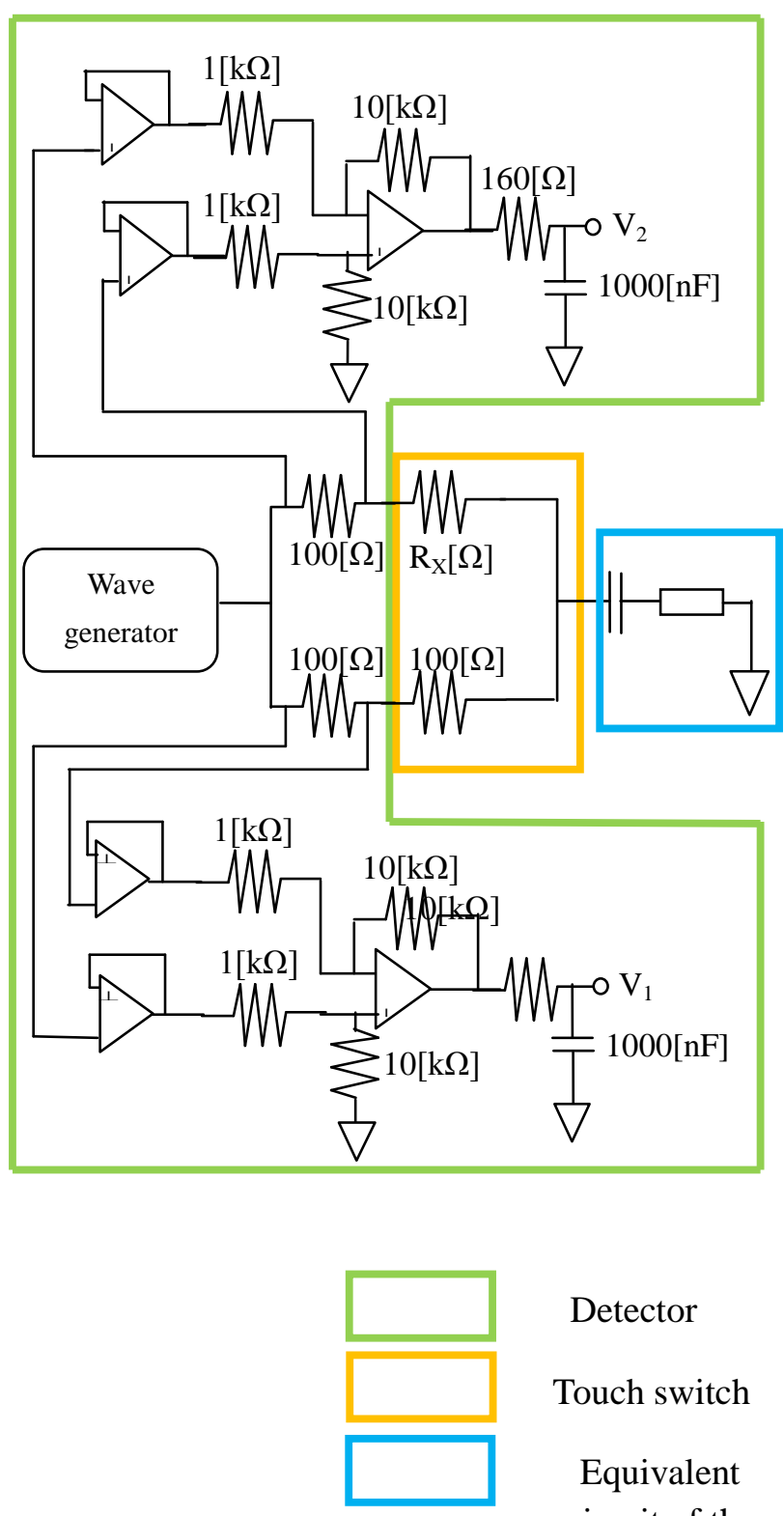

Detector

Touch switch

Equivalent circuit of the human body

Fig.2 Device organization

\subsection{Theoretical value}

The formula for the theoretical value by the number of touch switches will be different. I describe the expression of the theoretical value in each case.

\subsubsection{If one is a touch switch}

Touch switch is shown in Fig. 3 a block diagram of one case. And $i_{1}, i_{2}$ respectively, the current flowing through the resistance of the detector, and $\mathrm{V}$ the voltage across the switch and the touch detection unit. When each of these, $i_{2}$ and $i_{1}$ current becomes formula (1) and formula (2). 


$$
\begin{aligned}
& i_{l}=\frac{R_{1}}{R_{1}+R_{2}} V \\
& i_{2}=\frac{R_{1}}{R_{1}+R_{x}} V
\end{aligned}
$$

and the current ratio calculated from the formula (1) and formula (2),

$$
\frac{i_{2}}{i_{1}}=\frac{2 R_{1}}{R_{1}+R_{x}}
$$

The current ratio is determined by the value of the resistance, regardless of the voltage.

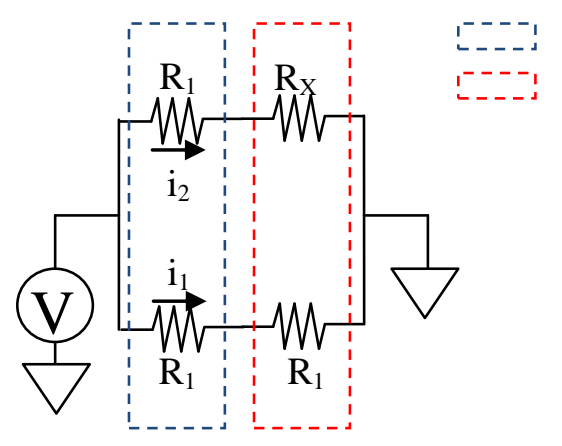

Fig.3 Touch Switch is a block diagram of a single case

\subsubsection{If a plurality of touch switches}

Touch switch shows a block diagram of a plurality of the case Fig.4. The case of Fig.4, the finger is touching the person of one touch switch. The current flows to the ground through a human body by finger touches the touch switch. However, the configuration of the circuit, such as the form of the bridge circuit current also flows toward the touch switch the finger does not touch all of the current is not flowing. Therefore, if a plurality of touch switches can not be calculated theoretical theoretical formula obtained in 2.2.1.Accordingly, determined using Kirchhoff's law expression when a plurality of touch switches theoretical.

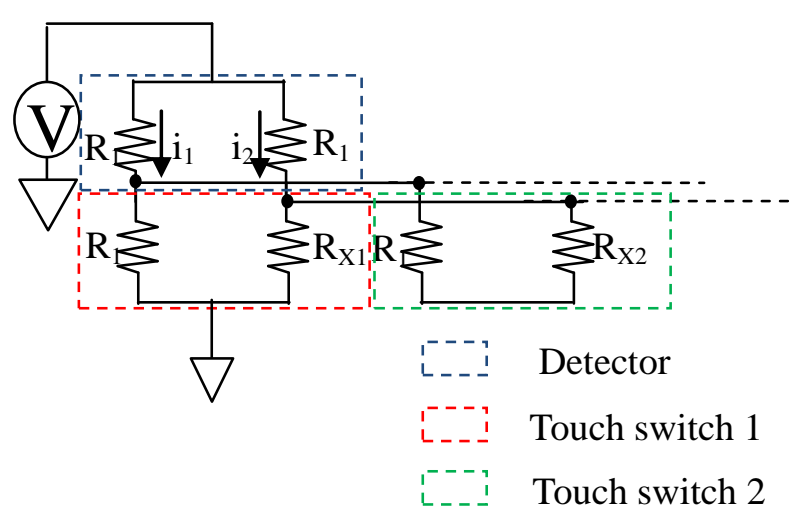

Fig.4 Touch Switch is a block diagram of a case of multiple

\subsection{2.(a) Method of calculating the theoretical value}

I show a bridge circuit representing Fig. 3 to Fig.4. There is more than one switch, G of Fig.5 is the combined resistance value of the resistor connected to the finger touch switch are not touching. Solving the equation in each of the three loops shown in Fig.10 equation (4), equation (5) becomes equation (6).

$$
\begin{aligned}
& R_{l}\left(i_{1}-i_{2}-i_{3}\right)+R_{l}\left(i_{1}-i_{3}\right)=V \\
& R_{l}\left(i_{2}+i_{3}\right)+G i_{2}+R_{l}\left(i_{2}+i_{3}-i_{1}\right)=0 \\
& R_{l}\left(i_{2}+i_{3}\right)+R_{X I} i_{3}+R_{l}\left(i_{3}-i_{1}\right)+R_{l}\left(i_{2}+i_{3}-i_{1}\right)=0
\end{aligned}
$$

By Equation (4), equation (5), equation (6), each of these, $i_{1}, i_{2}, i_{3}$ becomes formula $(7)$, formula $(8)$,formula (9).

$$
\begin{aligned}
i_{1}= & \frac{\left(2 R_{1}+G\right)\left(R_{1}+R_{X 1}\right)}{R_{1}\left\{R_{1}\left(R_{1}+3 R_{X 1}\right)+G\left(R_{1}+R_{X 1}\right)\right\}} V \\
i 2= & \frac{R_{X 1}-R_{1}}{R_{1}\left(R_{1}+3 R_{X 1}\right)+G\left(R_{1}+R_{X 1}\right)} V \\
i 3= & \frac{2 R_{1}+G}{R_{1}\left(R_{1}+3 R_{X 1}\right)+G\left(R_{1}+R_{X 1}\right)} V
\end{aligned}
$$

Further, in Fig.4, if you $\mathrm{I}_{1}, \mathrm{I}_{2}$, respectively the current flowing through the detector, $\mathrm{I}_{1}, \mathrm{I}_{2}$ is expressed formula (10), formula (11).

$$
\begin{aligned}
& I_{1}=i_{1}-i_{2}-i_{3} \\
& I_{2}=i_{2}+i_{3}
\end{aligned}
$$

and substituting a i1, i2, i3 of formula (7) formula (8), formula (9) to formula (10), formula (11),

$$
I_{1}=\frac{\left(2 R_{1}+G\right)\left(R_{1}+R_{X 1}\right)-R_{1}\left(R_{1}+R_{X 1}+G\right)}{R_{1}\left\{R_{1}\left(R_{1}+3 R_{X 1}\right)+G\left(R_{1}+R_{X 1}\right)\right\}} V
$$




$$
I_{2}=\frac{R_{1}\left(R_{1}+R_{X 1}+G\right)}{R_{1}\left\{R_{1}\left(R_{1}+3 R_{X 1}\right)+G\left(R_{1}+R_{X 1}\right)\right\}} V
$$

and determining the current ratio formula (12), formula (13)

$$
\frac{I_{2}}{I_{1}}=\frac{R_{1}\left(R_{1}+R_{X 1}+G\right)}{\left(2 R_{1}+G\right)\left(R_{1}+R_{X 1}\right)-R_{1}\left(R_{1}+R_{X 1}+G\right)}
$$

Similarly becomes equation (3), the current ratio is determined by the value of the voltage regardless of the resistance. From the theoretical formula 2.2.1 and 2.2.2, the current ratio is determined by the magnitude of resistance regardless of the way the human touch touching the touch switch Moreover, the magnitude of the voltage of the impedance of the human body and a person has.

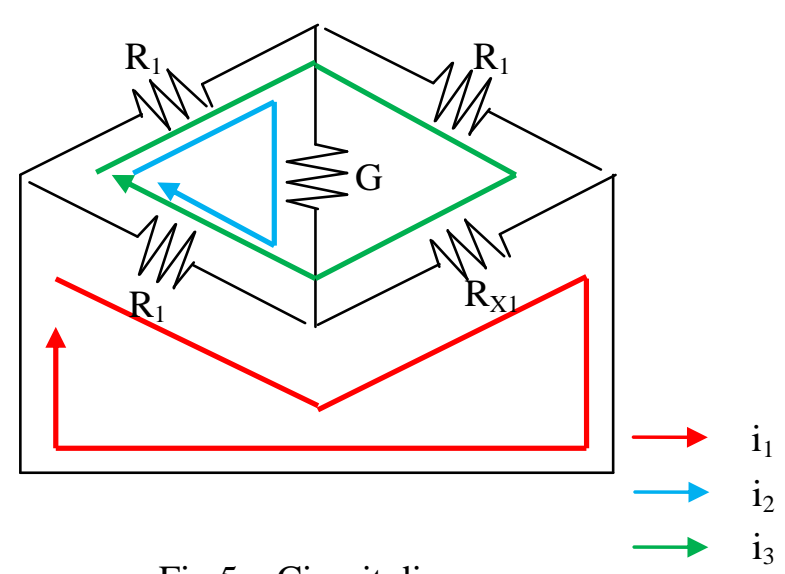

Fig.5 Circuit diagram

\subsection{2.(b) Calculation of the combined resistance value $G$}

Touch switch is a block diagram of the three in the case of Fig.6. When you use more than one touch switch, Fig.6 is the same as the block diagram as shown in Fig.7 because it is connected in parallel to each of the touch switch. G is the combined resistance of the touch switch touch switch 2 and 3 Assuming in Fig.7, and touches the touch switch 1. And G1, G2, respectively, the sum of the resistance touch switch 2 and touch switch 3. Formula G1, $\mathrm{G} 2$ are as in formula (15) and formula (16).

$$
\begin{aligned}
& G_{1}=R_{I}+R_{X 2} \\
& G_{2}=R_{I}+R_{X 3}
\end{aligned}
$$

Since G in Fig.7 is a parallel circuit of formula G1 and $\mathrm{G} 2$ where $\mathrm{G}$ is as in (17).

$$
G=\frac{G_{1} G_{2}}{G_{1}+G_{2}}
$$

In addition, substituting the G1, G2 of formula (15) and formula (16) to formula (17), possible to calculate the $\mathrm{G}$ can be represented by the formula (18).

$$
G=\frac{\left(R_{1}+R_{X 2}\right)\left(R_{1}+R_{X 3}\right)}{\left(R_{1}+R_{X 2}\right)+\left(R_{1}+R_{X 3}\right)}
$$

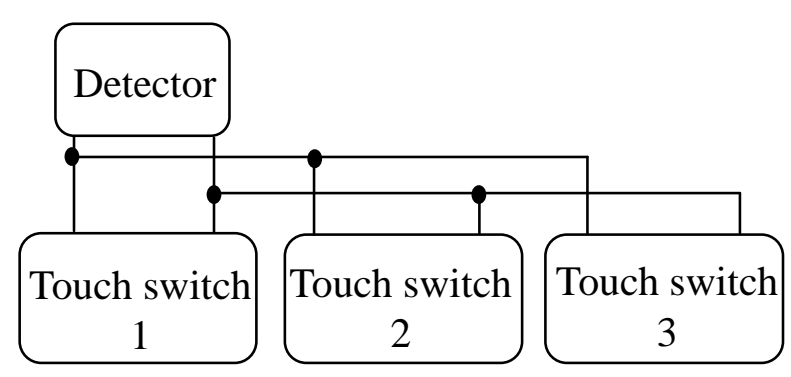

Fig.5 Touch switch is a block

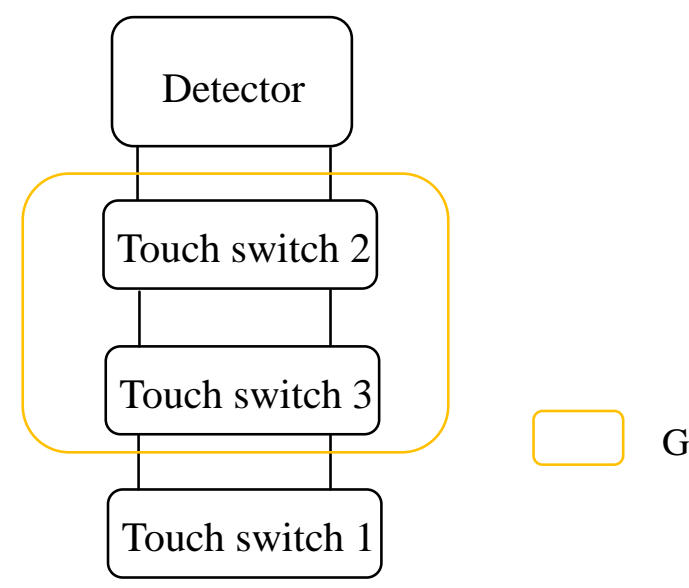

Fig.7 Touch switch is a block diagram of the three case 2

\section{Experiment}

\subsection{Experimental methodology}

Fig. 8 shows a block diagram of the circuit. In this study, the touch switch current ratio was measured up to 1-5. Then, the switch portion was set to a rectangle of various sizes using the aluminum foil as a metal film on the familiar. Also be applied to the detection of a sine wave oscillation frequency of 1 [MHz] from the function generator. Assuming that $\left(\mathrm{R}_{1}, \mathrm{R}_{\mathrm{X} 1}\right)$ a combination of the resistance of the touch switch shown in Fig.8, to $100[\Omega]$ the value of $R_{1}$, we have measured the current ratio varied from $100,180,270,360,470[\Omega]$ the value of $R_{X}$. In addition, this voltage ratio is equivalent to the current ratio by taking the 
ratio of $V_{1}, V_{2}$ as shown in Fig.8 so that the fixed $R_{1}=100$ $[\Omega]$ the resistance of the detector.

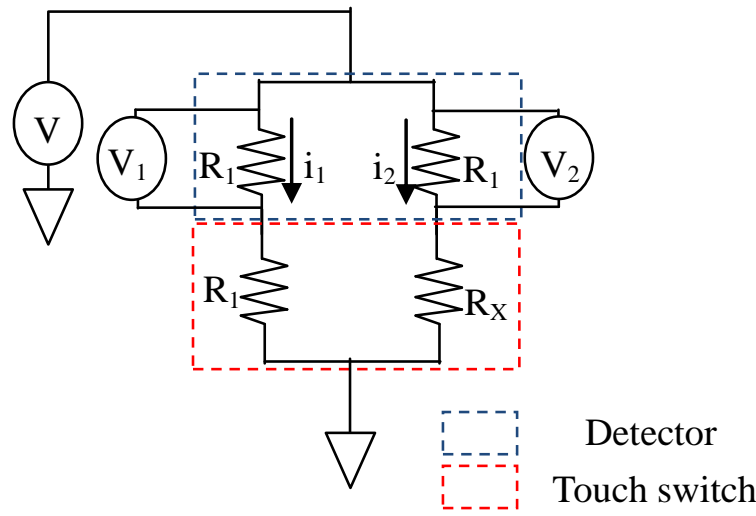

Fig.8 Diagram

\subsection{Experimental Results}

\subsubsection{If one is a touch switch}

Theoretical value is obtained by substituting each $\mathrm{R}_{1}=$ $100[\Omega]$, the $\mathrm{R}_{\mathrm{X}}=100,180,270,360,470[\Omega]$ in formula (29). And I find the relative error between the theoretical value and the measured value was determined. Measured value and the theoretical value, the graph summarizes the relative error of the measured value and the theoretical value, in Figure 9 in Title 1 and Title 2.

Title. 1 Touch switches when the measured value and the

\begin{tabular}{|c|c|c|c|c|c|}
\hline \multicolumn{7}{|c|}{ theoretical value of one } \\
\hline $\mathrm{R}_{\mathrm{X}}[\Omega]$ & 100 & 180 & 270 & 360 & 470 \\
\hline Measurements & 1 & 0.714 & 0.541 & 0.435 & 0.351 \\
\hline $\begin{array}{c}\text { Theoretical } \\
\text { value }\end{array}$ & 1 & 0.704 & 0.533 & 0.438 & 0.353 \\
\hline
\end{tabular}

Title.2 Touch Switch is the relative error in the case of one

\begin{tabular}{|c|c|c|c|c|c|}
\hline $\mathrm{R}_{\mathrm{X}}[\Omega]$ & 100 & 180 & 270 & 360 & 470 \\
\hline Error[\%] & 0 & 1.4 & 1.5 & 0.7 & 0.6 \\
\hline
\end{tabular}

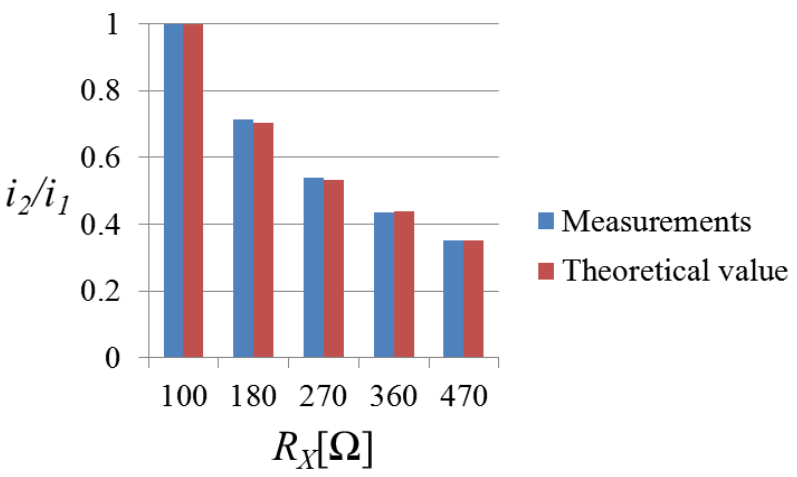

Fig.9 Touch switch experimental results in the case of one

\subsubsection{If a plurality of touch switches}

Theoretical value is obtained by substituting $\mathrm{R}_{1}=100$ $[\Omega]$, the $\mathrm{R}_{\mathrm{X}}=100,180,270,360,470[\Omega]$ in formula (40). And I find the relative error between the theoretical value and the measured value was determined. Summarizes the average value of the measured value and the theoretical value of the touch switches for each piece, are summarized in the chart. In addition, also put together a relative error in each piece.

Title. 3 Touch Switch is the average value of the measured value and the theoretical value at the time of two

\begin{tabular}{|c|c|c|c|c|c|}
\hline $\mathrm{R}_{\mathrm{X}}[\Omega]$ & 100 & 180 & 270 & 360 & 470 \\
\hline Measurements & 0.986 & 0.686 & 0.563 & 0.492 & 0.449 \\
\hline $\begin{array}{c}\text { Theoretical } \\
\text { value }\end{array}$ & 1 & 0.689 & 0.553 & 0.484 & 0.436 \\
\hline
\end{tabular}

Title.4 Touch Switch is the relative error in the case of two

\begin{tabular}{|c|c|c|c|c|c|}
\hline $\mathrm{R}_{\mathrm{X}}[\Omega]$ & 100 & 180 & 270 & 360 & 470 \\
\hline Error[\%] & 1.4 & 0.4 & 1.8 & 1.7 & 3 \\
\hline
\end{tabular}

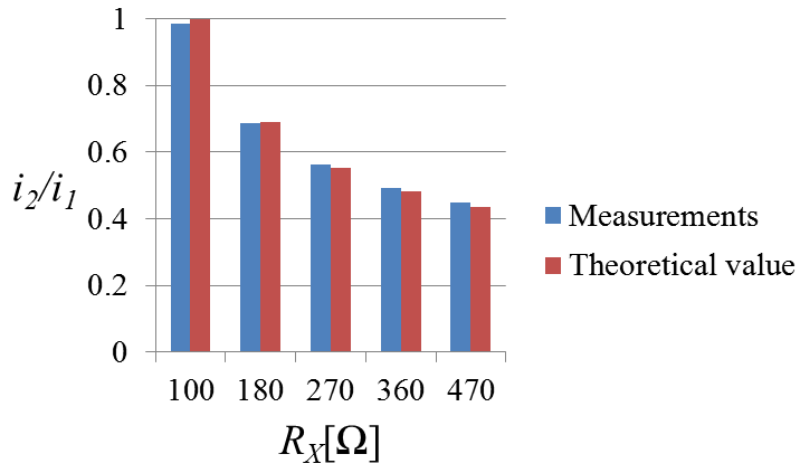

Fig.10 Touch switch experimental results in the case of two

Title.5 Touch Switch is the average value of the measured value and the theoretical value at the time of three 


\begin{tabular}{|c|c|c|c|c|c|}
\hline $\mathrm{R}_{\mathrm{X}}[\Omega]$ & 100 & 180 & 270 & 360 & 470 \\
\hline Measurements & 0.978 & 0.778 & 0.646 & 0.608 & 0.568 \\
\hline $\begin{array}{c}\text { Theoretical } \\
\text { value }\end{array}$ & 1 & 0.763 & 0.656 & 0.599 & 0.563 \\
\hline
\end{tabular}

Title.6 Touch Switch is the relative error in the case of three

\begin{tabular}{|c|c|c|c|c|c|}
\hline $\mathrm{R}_{\mathrm{X}}[\Omega]$ & 100 & 180 & 270 & 360 & 470 \\
\hline Error[\%] & 2.2 & 2 & 1.5 & 1.5 & 0.9 \\
\hline
\end{tabular}

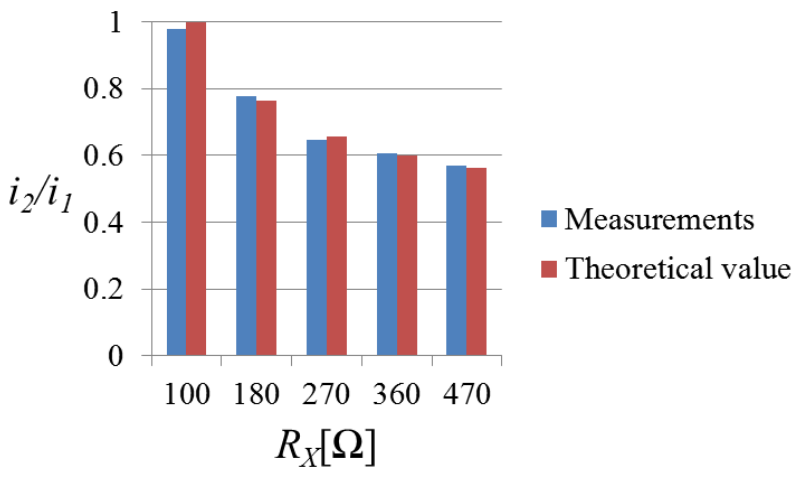

Fig.11 Touch switch experimental results in the case of three

Title.7 Touch Switch is the average value of the measured value and the theoretical value at the time of four

\begin{tabular}{|c|c|c|c|c|c|}
\hline $\mathrm{R}_{\mathrm{X}}[\Omega]$ & 100 & 180 & 270 & 360 & 470 \\
\hline Measurements & 0.981 & 0.797 & 0.716 & 0.688 & 0.647 \\
\hline $\begin{array}{c}\text { Theoretical } \\
\text { value }\end{array}$ & 1 & 0.81 & 0.727 & 0.677 & 0.645 \\
\hline
\end{tabular}

Title. 8 Touch Switch is the relative error in the case of four

\begin{tabular}{|c|c|c|c|c|c|}
\hline $\mathrm{R}_{\mathrm{X}}[\Omega]$ & 100 & 180 & 270 & 360 & 470 \\
\hline Error[\%] & 1.9 & 1.6 & 1.6 & 1.6 & 0.3 \\
\hline
\end{tabular}

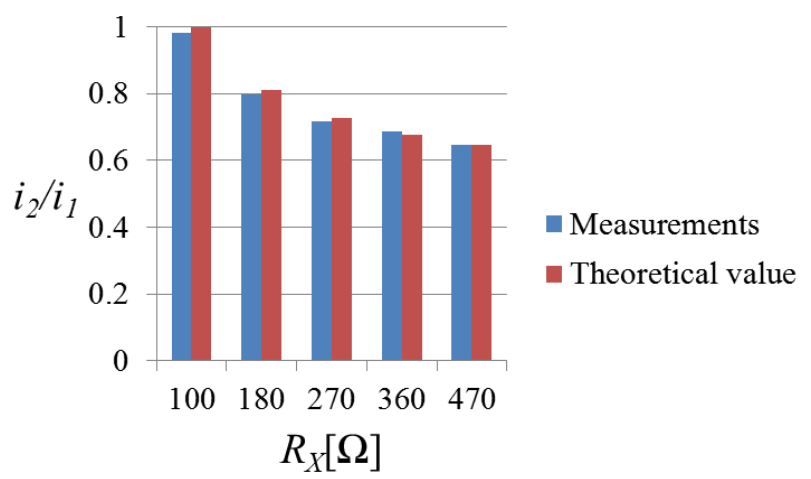

Fig.12 Touch switch experimental results in the case of four
Title.9 Touch Switch is the average value of the measured value and the theoretical value at the time of five

\begin{tabular}{|c|c|c|c|c|c|}
\hline $\mathrm{R}_{\mathrm{X}}[\Omega]$ & 100 & 180 & 270 & 360 & 470 \\
\hline Measurements & 1 & 0.853 & 0.743 & 0.731 & 0.705 \\
\hline $\begin{array}{c}\text { Theoretical } \\
\text { value }\end{array}$ & 1 & 0.842 & 0.768 & 0.729 & 0.701 \\
\hline
\end{tabular}

Title.10 Touch Switch is the relative error in the case of five

\begin{tabular}{|c|c|c|c|c|c|}
\hline $\mathrm{R}_{\mathrm{X}}[\Omega]$ & 100 & 180 & 270 & 360 & 470 \\
\hline Error[\%] & 0 & 1.3 & 3.3 & 0.3 & 0.6 \\
\hline
\end{tabular}

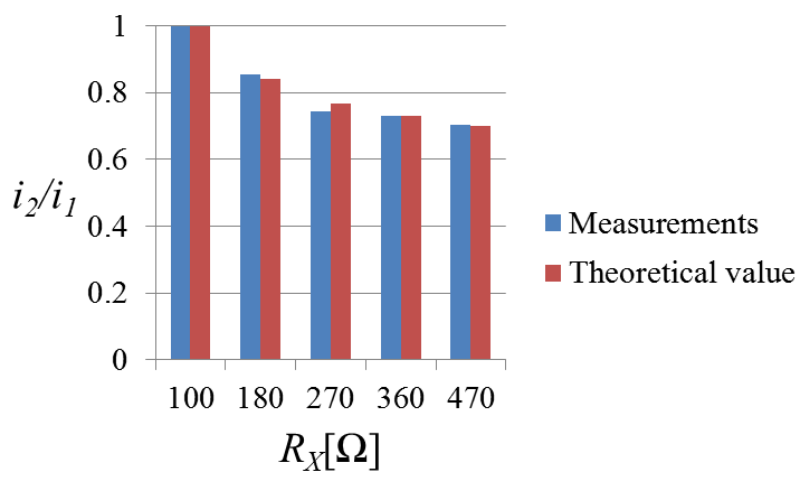

Fig.13 Touch switch experimental results in the case of five

\section{Conclusion}

In this paper,form and arrangement are the proposals of the touch switch which can be changed arbitrarily. I below shows the results obtained in this study.

- Due to the difference of the current ratio, the identification of the touch switches can be

- Current ratio is determined by the resistance value does not depend on the shape of the metal film of the switch

- Can be used as a touch switch at various locations in a plane is not limited

Showed that by this method make a shape and arrangement of the touch switch can be changed arbitrarily. Therefore, miniaturization of the device is expected, it is to be used in places like irregularities or spherical. It is also possible to create a panel with my applications requiring only made possible by a combination of various touch switch array, the addition, the touch panel can be shaped like. 


\section{References}

(1) Krein : "The electroquasistatics of the capacitive touch panel", IEEE Transactions on,Volume26, pp.529-534, Japan,2013.

(2) Changhong Jiang: "The control system of the Blind Hole Processing Machine based on PLC and touch-panel" Conference Publications, Volume4, pp. 556- 558,Japan, 2013.

(3) Asano, K:" Transparent touch panel with conductive liquid channel structure", Conference Publications, pp.1022-1025,Japan,2013. 